\title{
Advanced materials for energy harvesting, storage, sensing and environmental engineering $\|^{\star}$
}

\author{
Mohammed El Ganaoui ${ }^{1}$, Mohamed El Jouad ${ }^{2}$, Rachid Bennacer ${ }^{3}$, and Jean-Michel Nunzi ${ }^{4, *}$ \\ ${ }^{1}$ University of Lorraine, Lab Lermab, IUT Longwy, 186 Rue Lorraine, Longwy F-54400, France \\ ${ }^{2}$ Chouaib Doukkali University, National School of Applied Science, Science Engineer Laboratory for Energy (LabSIPE), \\ El Jadida, Morocco \\ ${ }^{3}$ LMT/ENS-Paris-Saclay/CNRS/Université Paris Saclay, 4 Avenue des Sciences 91190 Gif sur Yvette, France \\ ${ }^{4}$ Queens University, Department of Chemistry, Department of Physics, Engineering Physics \& Astronomy, Kingston ON K7L \\ 3N6, Canada
}

Received: 27 November 2020 / Accepted: 3 December 2020

During 2020, the world's attention turned towards Science more than ever, searching for answers and solutions. That is an important indication of the collective belief into demonstration and proof, which permit to understand, evaluate, solve and predict the future, the evolution of life on a daily basis as well as to provide answers to events that are threatening humanity locally or globally.

Along these lines, scientific activity benefited massively this year from its past and present production in terms of communication and liaison. Let us remind that this environment in the broadest sense could not have come into reality without abstraction, mathematical tools, rigorous experimentation, advanced measurement and detection, which all together enable us to offer a clearer and unambiguous vision in an environment restricted by the filter of habits and feelings.

In the context of the 2019-2020 world health crisis (namely COVID 19), we have become more aware of the usefulness and importance of biosensors [1-3]. From an energy point of view (production and storage), this health crisis was accompanied by a drop in the demand and prices for energy. As a result, several countries have reduced their energy bills and become more dependent on the international market. This upheaval in the producer-consumer balance will leave its mark. It also underlines the importance of working on the diversification of the energy mix, and in particular on the efficiency of production and storage of renewable energies [4-9].

This Topical issue to the European Physical Journal Applied Physics on Materials for Energy Harvesting, Storage, Sensing and Environmental Engineering intends to dress an updated and equilibrated picture of the multidisciplinary connections between energy and man-

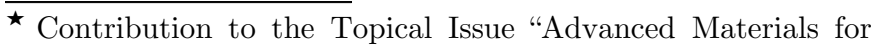
Energy Harvesting, Storage, Sensing and Environmental Engineering (ICOME 2019)", edited by Mohammed El Ganaoui, Mohamed El Jouad, Rachid Bennacer, Jean-Michel Nunzi.

* e-mail: nunzijm@queensu.ca
}

agement, as previous issues did [10-13]. This year's issue covers multidisciplinary areas of applied research into which Physics has a say: energy transfer, conversion and storage [14-17], fluid mechanics and microfluidics [18,19], materials [20-22]. Modelling and simulation complement the issue by providing understanding and prediction to experimental situations, with various approaches at multiple scales: nano, micro, meso and macroscopic [23-27].

L'attention du monde s'est tournée en 2020, plus que jamais vers la science, à la recherche de réponses et de solutions. C'est une indication importante de la croyance collective en démonstration et preuve, qui permettent de comprendre, d'évaluer, de résoudre et de prédire l'avenir, l'évolution de la vie au quotidien ainsi que de fournir des réponses aux événements qui menacent l'humanité localement ou globalement.

Dans ce sens, l'activité scientifique a massivement bénéficié cette année de sa production passée et présente en termes de communication et de liaison. Rappelons que cet environnement au sens large n'aurait pu se concrétiser sans abstraction, outils mathématiques, expérimentation rigoureuse, mesure et détection avancées, qui tous ensemble nous permettent d'offrir une vision plus claire et sans ambiguïté dans un environnement restreint par le filtre des habitudes et des sentiments.

Dans le contexte de la crise sanitaire mondiale 2019 2020 (à savoir le COVID 19), nous avons pris davantage conscience de l'utilité et de l'importance des biocapteurs [1-3]. Du point de vue énergétique (production et stockage), cette crise sanitaire s'est accompagnée d'une baisse de la demande et des prix de l'énergie. En conséquence, plusieurs pays ont réduit leurs factures d'énergie et sont devenus plus dépendants du marché international. Ce bouleversement de l'équilibre producteur-consommateur laissera sa marque. Il souligne aussi l'importance de travailler sur la diversification du mix énergétique, et notamment sur l'efficacité de la production et du stockage des énergies renouvelables [4-9]. 
Ce numéro spécial de l'European Physical Journal Applied Physics sur les matériaux pour la production d'énergie, son stockage, les capteurs et l'ingénierie de l'environnement entend dresser un tableau actuel et équilibré des liens multidisciplinaires entre énergie et gestion, dans la lignée des numéros précédents [10-13]. Le numéro de cette année couvre des domaines multidisciplinaires de recherche appliquée dans lesquels la physique a son mot à dire: transfert, conversion et stockage d'énergie [14-17], mécanique des fluides et microfluidique [18,19], matériaux [20-22]. La modélisation et la simulation complètent la problématique en permettant de comprendre et de prédire les situations expérimentales, avec différentes approches à plusieurs échelles: nano, micro, méso et macroscopique [23-27].

\section{References}

1. S. Lee, H. Han, C.-H. Kim, Eur. Phys. J. Appl. Phys. 91, $20201(2020)$

2. R. Samson, G.R. Navale, M.S. Dharne, 3 Biotech 10, 385 (2020)

3. B.V. Ribeiro, T.A.R. Cordeiro, G.R.O. e Freitas, L.F. Ferreira, D.L. Franco, Talanta Open 2, 100007 (2020)

4. D. Salem, M. Shalabi, F. Souissi, F. Nemmar, M. Said Belkaid, M. Aamir, J.M. Nunzi, Eur. Phys. J. Appl. Phys. 90, 30201 (2020)

5. U. Jabeen, T. Adhikari, S. Mujtaba Shah, D. Pathak, T. Wagner, J.M. Nunzi, Eur. Phys. J. Appl. Phys. 78, 34809 (2017)

6. U. Jabeen, T. Adhikari, S. Mujtaba Shah, D. Pathak, T. Wagner, J.M. Nunzi, Eur. Phys. J. Appl. Phys. 78, 34811 (2017)

7. U. Jabeen, T. Adhikari, S. Mujtaba Shah, J.M. Nunzi, Eur. Phys. J. Appl. Phys. 78, 34810 (2017)

8. J. Baffreau, S. Leroy-Lhez, H. Derbal, A.R. Inigo, J.-M Nunzi, M.M. Groeneveld, R.M. Williams, P. Hudhomme, Eur. Phys. J. Appl. Phys. 36, 301 (2006)

9. L. Sicot, C. Fiorini, A. Lorin, P. Raimond, C. Sentein, J.M. Nunzi, Sol. Energy Mater. Sol. Cells 63, 49 (2000)
10. R. Bennacer, M. El Ganaoui, J.M. Nunzi, Eur. Phys. J. Appl. Phys. 74, 24601 (2016)

11. M. El Ganaoui, J.-M. Nunzi, R. Bennacer, Eur. Phys. J. Appl. Phys. 78, 34801 (2017)

12. J.M. Nunzi, R. Bennacer, M. El Ganaoui, Eur. Phys. J. Appl. Phys. 85, 20901 (2019)

13. M. El Jouad, R. Bennacer, M. El Ganaoui, J.-M Nunzi, Eur. Phys. J. Appl. Phys. 88, 20903 (2019)

14. S. Bhattacharyya, D. Sarkar, U. Shettar Mahabaleshwar, M. K. Soni, M. Mohanraj, Eur. Phys. J. Appl. Phys. 92, 30901 (2020)

15. A. Labrag, M. Bghour, A. Abou El Hassan, H. El Hamidi, A. Taoufik, S. Laasri, Eur. Phys. J. Appl. Phys. 92, 20601 (2020)

16. B. Liu, L. Chai, A. Chen, F. Tang, K. Sefiane, G.E.I. Achkar, Eur. Phys. J. Appl. Phys. 86, 30902 (2019)

17. A. Alaoui-Belghiti, M. Rkhis, S. Laasri, A. Hajjaji, M. Eljouad, R. EL-Otmani, E.-K. Hlil, Eur. Phys. J. Appl. Phys. 87, 20902 (2019)

18. R. Slimani, A. Aissa, F. Mebarek-Oudina, U. Khan, M. Sahnoun, A.J. Chamkha, M.A. Medebber, Eur. Phys. J. Appl. Phys. 92, 10904 (2020)

19. M. Driouche, T. Rezoug, M. El Ganaoui, Eur. Phys. J. Appl. Phys. 88, 20901 (2019)

20. A. Alaoui-Belghiti, Y. Tabbai, M. Rkhis, S. Laasri, H. Lifi, A. Laghzizil, K. Strzałkowski, A. Hajjaji, Eur. Phys. J. Appl. Phys. 92, 10902 (2020)

21. S. El Asri, H. Ahamdane, L. Hajji, M. El Hadri, M. Ahmed El Idrissi Raghni, M. Mansori, Eur. Phys. J. Appl. Phys. 92, 10901 (2020)

22. B. Liu, S.-W. Wang, L. Chai, G. El Achkar, A. Chen, P.E. Theodorakis. Eur. Phys. J. Appl. Phys. 92, 11101 (2020)

23. A. Chen, X. Chen, X. Miao, J. Yin, B. Liu, Eur. Phys. J. Appl. Phys. 91, 31102 (2020)

24. R. Temsamani, A. Talbi, A. Mrigal, M. Addou, K. Nouneh, K. Gueddouch, H. Zaari, Eur. Phys. J. Appl. Phys. 92, 10302 (2020)

25. Z. Hireche, L. Nasseri, D. Eddine Ameziani, Eur. Phys. J. Appl. Phys. 91, 20904 (2020)

26. H. El Azrak, A. Hassani, K. Sbiaai, A. Hasnaoui, Eur. Phys. J. Appl. Phys. 91, 30301 (2020)

27. F. Eddiai, M. Dardouri, A. Hassani, M. Badawi, K. Sbiaai, A. Hassnaoui, Eur. Phys. J. Appl. Phys. 91, 31302 (2020)

Cite this article as: Mohammed El Ganaoui, Mohamed El Jouad, Rachid Bennacer, Jean-Michel Nunzi, Advanced materials for energy harvesting, storage, sensing and environmental engineering II, Eur. Phys. J. Appl. Phys. 93, 10902 (2021) 